\title{
Isospin violations at BESIII
}

\author{
Hongrong Qi**and Wencheng Yan \\ for the BESIII Collaboration \\ Beihang University, Beijing, 100083, People's Republic China \\ E-mail: Gihongrongebuaa.edu.cn, vanwcdbuaa.edu.cn
}

At present very large data samples in the energy region of 2.0-4.6 GeV were accumulated by the BESIII detector, which is operated in the upgraded Beijing electron positron collider (BEPCII). These data samples provide an unprecedented opportunity in the study of light hadron spectra and charmonium(-like) decays. We review some experimental analyses related to isospin violations at BESIII in this proceeding, which can be classify into three categories: isospin violating processes with a $f_{0}(980)$ or $a_{0}^{0}(980)$ production, isospin violating processes with baryon final states, and isospin violating hadronic transitions in the charmonium system.

XIII Quark Confinement and the Hadron Spectrum - Confinement 2018

31 July - 6 August 2018

Maynooth University, Ireland

\footnotetext{
* Speaker.

†qihongrong@buaa.edu.cn
} 


\section{Introduction}

Charmonium spectroscopy provides an ideal place to gain insights into the quark confinement and the decays of charmonium(-like) states. The isospin symmetry is conservative in strong interactions, while it could be violated in electromagnetic or weak interactions. The isospin violations will help us provide important probe of the relative amplitudes between strong interactions and electromagnetic interactions in charmonium decays, as well as the mass difference of the light $u$ and $d$ quarks.

The scalar meson $f_{0}(980)$, which is explained as a tetraquark [ [ a quark-antiquark gluon hybrid [ [ 9 ] in theory, is found with a width of $\sim 10 \mathrm{MeV}$ in isospin violating processes $[\mathbf{[}, \mathbf{6}, \mathbf{\square}]$, which is much narrower than that of $\sim 50 \mathrm{MeV}$ in the isospin conservation decays [ []]. The significant excess of $J / \psi \rightarrow \gamma \eta(1405) \rightarrow \gamma \pi^{0} f_{0}(980)$ with the anomalously large isospin violation was observed at BESIII [5]. A triangle singularity mechanism is proposed to interpret this unusual phenomenon [Q]. Another theory, which is thought to be a feasible approach to clarify the phenomenon of the narrower $f_{0}(980)$, is the mixing mechanism of $a_{0}^{0}(980)-f_{0}(980)[\mathbf{W}, \mathbf{W}, \mathbf{W}]$. The BESIII collaboration recently observed the $a_{0}^{0}(980)-f_{0}(980)$ mixing with the statistical significance in excess of 5.0 standard deviations $(\sigma)$ [ $\square]$.

Very recently, BESIII reported an evident difference in line shape and magnitude of the measured cross sections between $e^{+} e^{-} \rightarrow \Lambda(1520)\left(\rightarrow p K^{-}\right) \bar{n} K_{S}^{0}+$ c.c. and $e^{+} e^{-} \rightarrow p K^{-} \bar{\Lambda}(1520)(\rightarrow$ $\left.\bar{n} K_{S}^{0}\right)+$ c.c. [[2]]. We consider that such an isospin violating effect may be due to the interference between $I=1$ and $I=0$ final states. Other isospin violating processes with baryon final states, such as $J / \psi \rightarrow \Lambda \bar{\Lambda} \pi^{0}$ and $J / \psi \rightarrow \Lambda \bar{\Sigma}^{0}+c . c$, , are also reported.

The isospin violating hadronic transitions in the charmonium system play an essential probe of the isospin asymmetry mechanism. For this case, we investigated three processes at BESIII: $\psi^{\prime} \rightarrow$ $\pi^{0} J / \psi, \psi^{\prime} \rightarrow \pi^{0} h_{c}$, and $\chi_{c 0,2} \rightarrow \pi^{0} \eta_{c}$. In this proceeding, we also present the recently obtained results with isospin violations in so called exotic hadron decays at BESIII, such as $Y(4260) \rightarrow$ $\pi^{0} \eta J / \psi$ and $D_{s 0}^{*}(2317)^{ \pm} \rightarrow \pi^{0} D_{s}^{ \pm}$. In addition, the doubly OZI suppressed decay $J / \psi \rightarrow \pi^{0} \phi$ are also visited.

\section{Isospin violations with a $f_{0}(980)$ or $a_{0}^{0}(980)$ production}

The nature of the scalar states $f_{0}(980)$ and $a_{0}^{0}(980)$ have been controversial for several decades.

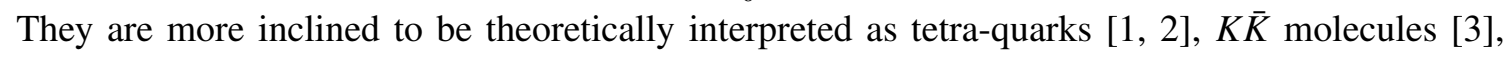
quark-antiquark gluon hybrids [四], etc., rather than conventional quark-antiquark mesons. Recent studies indicate that the isospin violating processes associated with the $f_{0}(980)$ or $a_{0}^{0}(980)$ would provide insights into the intrinsic nature of the unconventional mesons $f_{0}(980)$ and $a_{0}^{0}(980)$.

In 2012, a extremely narrow $f_{0}(980)$ signal with the width about $10 \mathrm{MeV}$ via $J / \psi \rightarrow \gamma \pi^{0} f_{0}(980)(\rightarrow$ $\pi^{+, 0} \pi^{-, 0}$ ) [四] was first observed, whereas its world average width is about $50-100 \mathrm{MeV} / c^{2}$ [[ए3] in that year. In that analysis, it is found that the $f_{0}(980)$ predominantly come from the $\eta(1405)$ decay, i.e., $\eta(1405) \rightarrow \pi^{0} f_{0}(980)$ [[]]. According to the measurement, the ratio $R=(\eta(1405) \rightarrow$ $\left.\left.f_{0}(980) \pi^{0}\right) \rightarrow \pi^{+} \pi^{-} \pi^{0}\right) /\left(\eta(1405) \rightarrow a_{0}^{0}(980) \pi^{0} \rightarrow \eta^{0} \pi^{0} \pi^{0}\right)$ is calculated to be $\left(17.9 \pm 4.2^{1}\right) \%$ [ [G],

\footnotetext{
${ }^{1}$ In the proceeding, the single uncertainty is the quadratic sum of the statistical item and the systematic one; if there are two uncertainties, the first one is statistical, and second systematic.
} 
which is a considerably large isospin violation. In theory, a model of triangle singularity Feynman diagram was proposed to reconcile the anomaly [Q].

In 2015, another $J / \psi \rightarrow V \pi^{0} f_{0}(980)$ measurement was observed at BESIII [ 6 ], where the vector particle $V$ is the $\phi$ meson. Similarly, the observed $f_{0}(980)$ is narrower (about $15 \mathrm{MeV}$ in width) than the world average in the same year [ [8]. It is strange that the significant $f_{1}(1285)$ instead of the $\eta(1405)$ is observed in the $\pi^{0} f_{0}(980)$ invariant mass spectrum, and the $f_{1}(1285) \rightarrow \pi^{0} f_{0}(980)$ is more clear with the statistical significance of $5.2 \sigma$ in the $f_{0}(980) \rightarrow \pi^{+} \pi^{-}$channel [5].

The mixing phenomenon in the $f_{0}(980)-a_{0}^{0}(980)$ system was first proposed in the late $1970 \mathrm{~s}$ and expected to shed light on the nature of these two unconventional mesons [س]]. Taking into account the difference between the $K^{+} K^{-}$and $K^{0} \bar{K}^{0}$ mass thresholds, the mixing mechanism predicted that a "resonant" peak (actually a cusp effect) appears with a width of about 10-20 MeV in the isospin violating reaction $\pi^{+} \pi^{-} \rightarrow \pi^{0} \eta$ [ए0], while the widths of both $f_{0}(980)$ and $a_{0}^{0}(980)$ are $50-100 \mathrm{MeV} / c^{2}$ [ [ [4] excluding in isospin violating decays. Later, theorists proposed to examine the $f_{0}(980)-a_{0}^{0}(980)$ mixing mechanism in the isospin violating decays $J / \psi \rightarrow \phi \eta \pi^{0}$ [ए5] and $\psi^{\prime} \rightarrow \gamma \chi_{c 1} \rightarrow \gamma \pi^{+} \pi^{-} \pi^{0}[\mathbb{W}]$. In addition, mixing intensities, $\xi_{f a}$ and $\xi_{a f}$, defined as effective experimental probes for the nature of $a_{0}^{0}(980)$ and $f_{0}(980)$, are sensitive to the coupling constants for $a_{0}^{0}(980) \rightarrow K \bar{K}$ and $f_{0}(980) \rightarrow K \bar{K}$, respectively.

In 2018, BESIII reported the observation of the $f_{0}(980)-a_{0}^{0}(980)$ mixing phenomenon in the decays of $J / \psi \rightarrow \phi \eta \pi^{0}\left(\eta \rightarrow \gamma \gamma\right.$ and $\left.\eta \rightarrow \pi^{+} \pi^{-} \pi^{0}, \phi \rightarrow K^{+} K^{-}\right)$and $\psi^{\prime} \rightarrow \gamma \chi_{c 1} \rightarrow \gamma \pi^{+} \pi^{-} \pi^{0}$ [四]. The excesses of $f_{0}(980)-a_{0}^{0}(980)$ mixing are determined with a statistical significance of larger than $5 \sigma$ for the first time, and the corresponding absolute branching fractions and mixing intensities are summarized in Table $\mathbb{W}$.

Table 1: The branching fractions $(\mathscr{B})$ and the intensities $(\xi)$ of the $f_{0}(980)-a_{0}^{0}(980)$ mixing. The first uncertainties are statistical, the second ones are systematic, and the third are related to parameterization of $\underline{f_{0}(980) \text { and } a_{0}^{0}(980) \text {. }}$

\begin{tabular}{lccc}
\hline \hline \multirow{2}{*}{ Channel } & \multicolumn{2}{c}{$f_{0}(980) \rightarrow a_{0}^{0}(980)$} & \\
& Solution I & Solution II & $a_{0}^{0}(980) \rightarrow f_{0}(980)$ \\
\hline $\mathscr{B}$ (mixing) $\left(10^{-6}\right)$ & $3.18 \pm 0.51 \pm 0.38 \pm 0.28$ & $1.31 \pm 0.41 \pm 0.39 \pm 0.43$ & $0.35 \pm 0.06 \pm 0.03 \pm 0.06$ \\
$\mathscr{B}(\mathrm{EM})\left(10^{-6}\right)$ & $3.25 \pm 1.08 \pm 1.08 \pm 1.12$ & $2.62 \pm 1.02 \pm 1.13 \pm 0.48$ & - \\
$\mathscr{B}$ (total) $\left(10^{-6}\right)$ & $4.93 \pm 1.01 \pm 0.96 \pm 1.09$ & $4.37 \pm 0.97 \pm 0.94 \pm 0.06$ & - \\
$\xi(\%)$ & $0.99 \pm 0.16 \pm 0.30 \pm 0.09$ & $0.41 \pm 0.13 \pm 0.17 \pm 0.13$ & $0.40 \pm 0.07 \pm 0.14 \pm 0.07$ \\
\hline \hline
\end{tabular}

We obtained the statistical significance of the mixing signal by scanning the two coupling constants $g_{a_{0} K^{+} K^{-}}$and $g_{f_{0} K^{+} K^{-}}$, which is depicted in Fig. W. For this two-dimensional distribution, the regions with higher statistical significance indicate larger probability for the emergence of the two coupling constants. The predicted coupling constants from various models [ए]] are displayed as well (color markers), but the theoretical uncertainties on the models are not considered here. Due to the limited statistics, a solid conclusion on internal structure of these two unconventional mesons is not yet drawn.

\section{Isospin violations in baryon final states}

Recently, BESIII reported that there is an evident difference in the line shape and magni- 


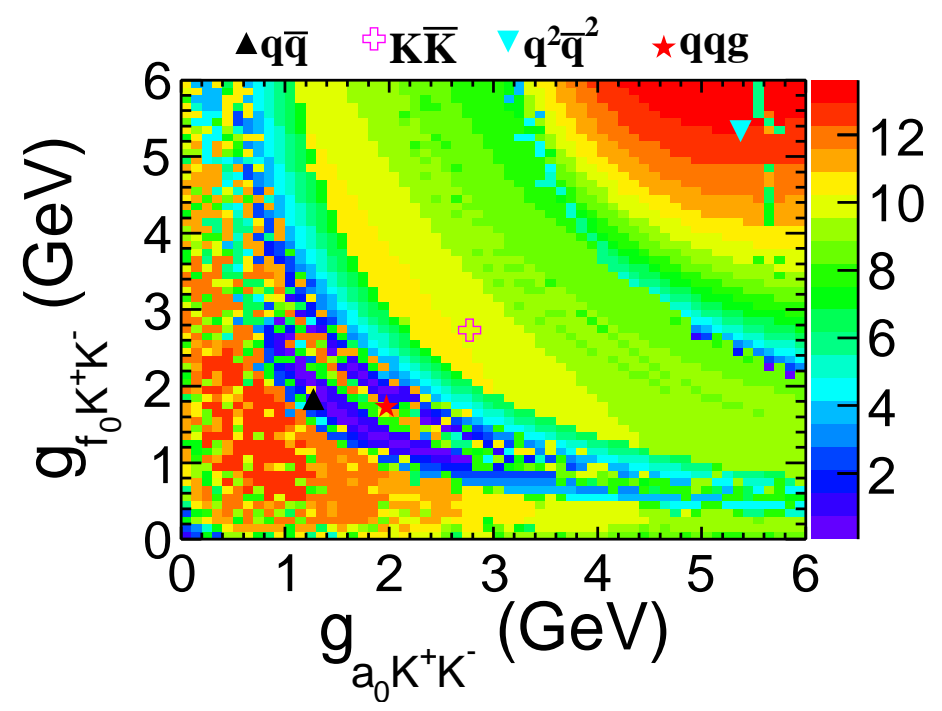

Figure 1: The statistical significance of the signal scanned in the two-dimensional space of $g_{a_{0} K^{+} K^{-}}$and $g_{f_{0} K^{+} K^{-}}$. The regions with higher statistical significance indicate larger probability for the emergence of the two coupling constants. The markers indicate predictions from various illustrative theoretical models.

tude of the measured Born cross sections between $e^{+} e^{-} \rightarrow \Lambda(1520)\left(\rightarrow p K^{-}\right) \bar{n} K_{S}^{02}$ and $e^{+} e^{-} \rightarrow$ $p K^{-} \bar{\Lambda}(1520)\left(\rightarrow \bar{n} K_{S}^{0}\right)$, as shown in Fig. [प[य]. The statistical significance of the cross-section difference is $3.1 \sigma$ at the c.m. energy of $3.770 \mathrm{GeV}$, while less than $3 \sigma$ at other energies due to lower statistics. Such an isospin violation effect is probably due to the interference between $I=1$ and

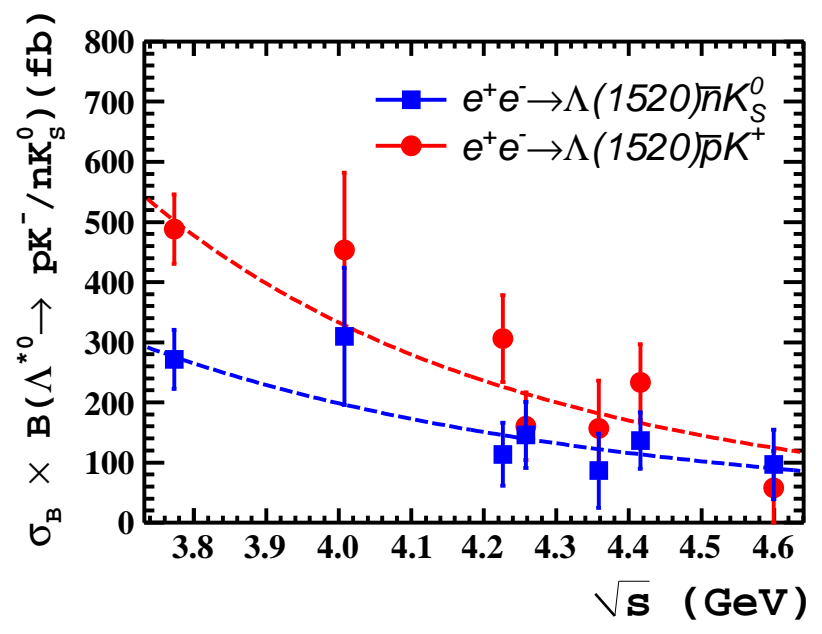

Figure 2: (Color online) Distributions of $\sigma_{\mathrm{B}}\left[e^{+} e^{-} \rightarrow \Lambda(1520) \bar{n} K_{S}^{0}\right] \times \mathscr{B}\left[\Lambda(1520) \rightarrow p K^{-}\right]$, and $\sigma_{\mathrm{B}}\left[e^{+} e^{-} \rightarrow\right.$ $\left.\Lambda(1520) \bar{p} K^{+}\right] \times \mathscr{B}\left[\Lambda(1520) \rightarrow n K_{S}^{0}\right]$ versus c.m. energy. The dots with error bars, which are the combined statistical and uncorrelated systematic uncertainties, represent data. The blue and red dashed lines are the fits to the cross sections of $e^{+} e^{-} \rightarrow \Lambda(1520) \bar{n} K_{S}^{0}$ and $e^{+} e^{-} \rightarrow \Lambda(1520) \bar{p} K^{+}$, respectively.

$I=0$ final states. The final states $\Lambda(1520) \bar{n} K_{S}^{0}$ and $\Lambda(1520) \bar{p} K^{+}$can be produced from $\bar{p} K^{+}$and

\footnotetext{
${ }^{2}$ In this section, charged conjugated modes are included unless otherwise indicated.
} 
$\bar{n} K_{S}^{0}$ systems either in $I=1$ or $I=0$ states, namely, excited $\bar{\Sigma}^{*}$ or $\bar{\Lambda}^{*}$ states. These two states can decay into both $\bar{p} K^{+}$and $\bar{n} K_{S}^{0}$ final states, but with a sign difference from $C G$ coefficients. Another possible approach is $e^{+} e^{-} \rightarrow \bar{K}^{*} K$ with the highly excited $\bar{K}^{*}$ decaying into $\Lambda(1520)\left(\rightarrow p K^{-}\right) \bar{n}$ or $\Lambda(1520)\left(\rightarrow n K_{S}^{0}\right) \bar{p}$. The $\bar{K}^{*} K$ system can be produced from $I=1\left(\right.$ excited $\left.\rho^{*}\right)$ or $I=0\left(\right.$ excited $\omega^{*}$ or $\left.\phi^{*}\right)$ states, where the interference effect can occur. If the final state is $p \bar{N}^{*}$ or $n \bar{N}^{*}$, a similar pattern could be observed. More experimental data are desirable to confirm these interpretations and speculations in the future. Theoretically, Ref. [ए]] claimed that the formalism associated with the electromagnetic form factors of the $\Lambda(1520)$ will shed light on the isospin violating cross sections if the measurement will be improved with higher precision.

In this proceeding, we present the recent measurements at BESIII of the branching fractions [ㅍ] ] of the isospin violating and isospin conserving decays of $J / \psi\left(\psi^{\prime}\right) \rightarrow \Lambda \bar{\Lambda} \pi^{0}$ and $J / \psi\left(\psi^{\prime}\right) \rightarrow$ $\Lambda \bar{\Lambda} \eta$, with drastically more precision than previous results [[13]]. The branching fractions in $J / \psi$ decays are determined to be $\mathscr{B}\left(J / \psi \rightarrow \Lambda \bar{\Lambda} \pi^{0}\right)=(3.78 \pm 0.27 \pm 0.30) \times 10^{-5}$ and $\mathscr{B}(J / \psi \rightarrow \Lambda \bar{\Lambda} \eta)=$ $(15.7 \pm 0.80 \pm 1.54) \times 10^{-5}$. Therefore, the ratio of $\mathscr{B}\left(J / \psi \rightarrow \Lambda \bar{\Lambda} \pi^{0}\right)$ to $\mathscr{B}(J / \psi \rightarrow \Lambda \bar{\Lambda} \eta)$ is equivalent to 0.24 , which indicates extremely large isospin violations. The branching fraction of $\psi^{\prime} \rightarrow \Lambda \bar{\Lambda} \eta$ is measured to be $(2.48 \pm 0.34 \pm 0.19) \times 10^{-5}$, while the upper limit of the branching fraction $\mathscr{B}\left(\psi^{\prime} \rightarrow \Lambda \bar{\Lambda} \pi^{0}\right)<0.29 \times 10^{-5}$ is obtained at the $90 \%$ confidence level. The ratio $R=\mathscr{B}\left(\psi^{\prime} \rightarrow \Lambda \bar{\Lambda} \pi^{0}\right) / \psi^{\prime} \rightarrow \Lambda \bar{\Lambda} \eta<0.12$

The branching fraction of the isospin symmetry breaking decay $J / \psi \rightarrow \Lambda \bar{\Sigma}^{0}$ will help elucidate the decay amplitude of $J / \psi \rightarrow B_{8} \bar{B}_{8}$ via three gluons or one photon [प18, [1]], and provide an insight into the SU(3)-flavour asymmetry [1, [2]] as well, where $B_{8}\left(\bar{B}_{8}\right)$ denotes an octet baryon (antibaryon). Using the sample of $(225.2 \pm 2.8) \times 10^{6} \mathrm{~J} / \psi$ events collected at BESIII, the branching fractions of $J / \psi \rightarrow \Lambda \bar{\Sigma}^{0}$ and $J / \psi \rightarrow \bar{\Lambda} \Sigma^{0}$ are measured to be $(1.46 \pm 0.11 \pm 0.12) \times 10^{-5}$ and $(1.37 \pm 0.12 \pm 0.11) \times 10^{-5}$, respectively [2]].

\section{Isospin violating hadronic transitions in the charmonium system}

The hadronic transitions $\psi^{\prime} \rightarrow \pi^{0}(\eta) J / \psi$ were suggested to be a reliable source for the extraction of the light quark mass ratio $m_{u} / m_{d}$, obtained by the equation [[22, [23],

$$
R_{\pi^{0} / \eta}=3\left(\frac{m_{d}-m_{u}}{m_{d}+m_{u}}\right) \frac{F_{\pi}^{2}}{F_{\eta}^{2}} \frac{M_{\pi}^{2}}{M_{\eta}^{2}}\left|\frac{\overrightarrow{q_{\pi}}}{\overrightarrow{q_{\eta}}}\right|^{3},
$$

where $R_{\pi^{0} / \eta}$ is the ratio of $\mathscr{B}\left(\psi^{\prime} \rightarrow \pi^{0} J / \psi\right)$ to $\mathscr{B}\left(\psi^{\prime} \rightarrow \eta J / \psi\right), F_{\pi(\eta)}$ and $M_{\pi(\eta)}$ are the decay constant and mass of the $\pi(\eta)$, respectively, $\vec{q}_{\pi(\eta)}$ stands for the momentum of the $\pi(\eta)$ in the rest frame of the $\psi^{\prime}$. Because the $J / \psi$ and $\psi^{\prime}$ are $\mathrm{SU}(3)$ singlets, it is obvious that the decay $\psi^{\prime} \rightarrow \pi^{0} J / \psi$ violates isospin symmetry, and the decay $\psi^{\prime} \rightarrow \eta J / \psi$ violates $\mathrm{SU}(3)$ flavor symmetry [24, [26]. Thereby, the decay amplitudes reflect the flavor symmetry breaking, and it is the mass differences within the multiplet that generates the isospin or the SU(3) breaking [R6]. The ratio $R_{\pi^{0} / \eta}$ was found to be $(3.74 \pm 0.06 \pm 0.04) \%$ by BESIII, with unprecedented high precision [27].

Another isospin-violating transition $\psi^{\prime} \rightarrow \pi^{0} h_{c}$ was observed to be $\mathscr{B}\left(\psi^{\prime} \rightarrow \pi^{0} h_{c}\right)=(8.4 \pm$ $1.3 \pm 1.0) \times 10^{-4}$ by the BESIII Collaboration [LE]. The measurements is consistent with a simple single-channel calculations based on the QCD multipole expansion using the Cornell potential model with predicting a partial width of $(0.12-0.36) \mathrm{KeV}$ [29]. 
BESIII Collaboration also search for the isospin-violating $\pi^{0}$ transition $\chi_{c 0,2} \rightarrow \pi^{0} \eta_{c}$, but only the upper limits on the branching fractions are determined at the $90 \%$ confidence level to be $\mathscr{B}\left(\chi_{c 0} \rightarrow \pi^{0} \eta_{c}\right)<1.6 \times 10^{-3}$ and $\left.\mathscr{B}\left(\chi_{c 2} \rightarrow \pi^{0} \eta_{c}\right)<3.2 \times 10^{-3}[B]\right]$.

\section{Other isospin violations at BESIII}

In recent years, BESIII reported a number of $X Y Z$ particles (also known as the charmoniumlike states) in the charmonium region. Some isospin violating processes associated with unconventional hadrons are searched for or observed, such as $Y(4260) \rightarrow \pi^{0} \eta J / \psi, D_{s 0}^{*}(2317)^{ \pm} \rightarrow \pi^{0} D_{s}^{ \pm}$. The tetraquark model [B] predicts that $Z_{c}(39000)$ can be produced in $Y(4260) \rightarrow \pi^{0} \eta J / \psi$ with $Z_{c}(3900)$ decaying into $\pi^{0} J / \psi$ and possibly $\eta J / \psi$ in the presence of sizable isospin violation. The molecular model [32] predicts a peak in the cross section of $Y(4260) \rightarrow \pi^{0} \eta J / \psi$ at the $D \bar{D}_{1}$ threshold, and a narrow peak in the $\eta J / \psi$ invariant mass spectrum at the $D \bar{D}^{*}$ threshold. BESIII performed the isospin violating process $Y(4260) \rightarrow \pi^{0} \eta J / \psi$, but no signal excess in either the $\eta J / \psi$ mass spectrum or the cross-section line shape [33]. The upper limit on the ratio of the branching fractions $\left(Y(4260) \rightarrow \pi^{0} \eta J / \psi\right) /\left(Y(4260) \rightarrow \pi^{0} \pi^{0} J / \psi\right)$ at the $90 \%$ confidence level is 0.15 at $\sqrt{s}=4.226 \mathrm{GeV}$ and 0.65 at $\sqrt{s}=4.257 \mathrm{GeV}$ [B3] .

The $D_{s 0}^{*}(2317)^{+}$meson is generally thought to be the P-wave $c \bar{s}$ state with spin-parity $J^{P}=0^{+}$. With a mass that is $45 \mathrm{MeV} / c^{2}$ below the $D K$ mass threshold, it is proposed as a $D K$ molecule [34]], a $c \bar{s} q \bar{q}$ tetraquark state [BS]], one of the chiral charmed doublets [B]], or a mixture of a $c \bar{s}$ meson and a $c \bar{s} q \bar{q}$ tetraquark [B]]. BESIII observed the $D_{s 0}^{*}(2317)^{ \pm}$in the decays $D_{s 0}^{*}(2317)^{ \pm} \rightarrow \pi^{0} D_{s}^{ \pm}$, and the absolute branching fraction is measured to be $\mathscr{B}\left(D_{s 0}^{*}(2317)^{ \pm} \rightarrow \pi^{0} D_{s}^{ \pm}\right)=1.00_{-0.14-0.14}^{+0.00+0.00}$ for the first time [B]8].

In addition, the doubly OZI suppressed decay $J / \psi \rightarrow \pi^{0} \phi$ are also investigated at BESIII. Considering the interference between $J / \psi \rightarrow \pi^{0} \phi$ and $J / \psi \rightarrow \pi^{0} K^{+} K^{-}$, the branching fraction $J / \psi \rightarrow \pi^{0} \phi$ is determined as $(2.94 \pm 0.16 \pm 0.16) \times 10^{-6}$ for constructive interference and $(1.24 \pm$ $0.33 \pm 0.30) \times 10^{-7}$ for destructive interference [B]]

\section{Summary}

In generally, there are two possible sources of isospin symmetry breaking, namely the electromagnetic processes and the difference between the masses of up and down quarks. Of late years, in order to interpret the phenomena with large isospin violations, a mixing mechanism and a triangle singularity diagram were proposed. In these proceeding, we summarized the isospin violating measurements in recent BESIII analyses with respect to these theories, which are mainly divided into three categories: isospin violating processes with a $f_{0}(980)$ or $a_{0}^{0}(980)$ production, isospin violations with baryon final states, and isospin violating hadronic transitions in the charmonium system.

\section{References}

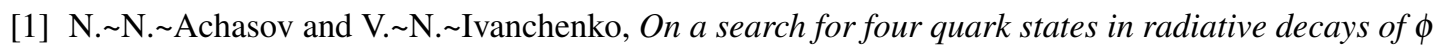
meson, Nucl. Phys. B315(1989) 465. 


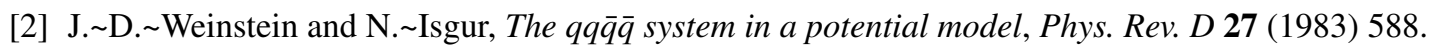

[3] J. D. Weinstein and N. Isgur, KK molecules, Phys. Rev. D 41 (1990) 2236.

[4] S. Ishida et al., in Proceedings of the 6th International Conference on Hadron Spectroscopy, Manchester, United Kingdom, 1995 (World Scientific, Singapore, 1995), p.454-456.

[5] M. Ablikim et al. (BESIII Collaboration), First observation of $\eta(1405)$ decays into $f_{0}(980) \pi^{0}$, Phys. Rev. Lett. $108(2012) 182001$.

[6] M. Ablikim et al. (BESIIICollaboration), Observation of the isospin violating decay

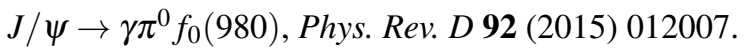

[7] M. Ablikim et al. (ESIII Collaboration), Observation of $a_{0}^{0}(980)-f_{0}(980)$ mixing, Phys. Rev. Lett. 121 (2018)022001.

[8] [. A. O Olive et al. (Particle Data Group), Review of Particle Physics, Chin. Phys. C 38 (2014) 090001

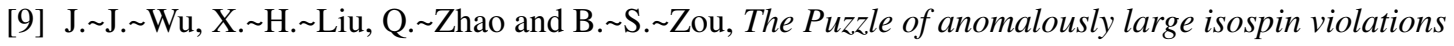
in $\eta(1405 / 1475) \rightarrow 3 \pi$, Phys. Rev. Lett. 108 (2012) 081803.

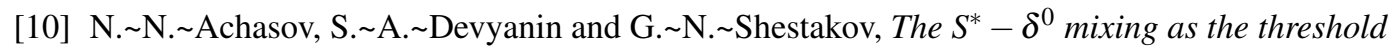
phenomenon, Phys. Lett. B 88(1979) 367.

[11] J. J. $\sim \mathrm{Wu}$ and B. $\sim$ S. ZZou, Study $a_{0}^{0}(980)-f_{0}(980)$ mixing from $a_{0}^{0}(980) \rightarrow f_{0}(980)$ transition, Phys. Rev. D 78(2008) 074017.

[12] M. Ablikim et al. (BESIII Collaboration), First measurement of $e^{+} e^{-} \rightarrow p K_{S}^{0} \bar{n} K^{-}+$c.c. above open charm threshold, Phys. Rev. D 98 (2018) 032014.

[13] ]. Beringer et al. (Particle Data Group), Review of Particle Physics (RPP), Phys. Rev. D 86 (2012) एण00ा

[14] M. Tanabashi et al. (Particle Data Group), Review of Particle Physics, Phys. Rev. D 98 (2018) D3000

[15] J. J. Wu, Q. Zhao and B. S. $\sim$ Zou, Possibility of measuring $a_{0}^{0}(980)-f_{0}(980)$ mixing from $\bar{V} / \psi \rightarrow \phi a_{0}^{0}(980)$, Phys. Rev. D 75 (2007) 114012.

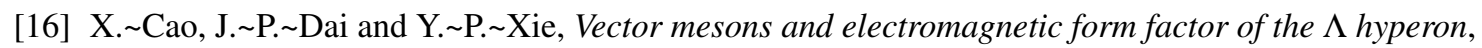
Phys. Rev. D 98 (2018)094006.

[17] M. Ablikim et al. (BESIII Collaboration), Measurements of the branching fractions for $J / \psi$ and $\psi^{\prime} \rightarrow \Lambda \Lambda \pi^{0}$ and $\Lambda \Lambda \eta$, Phys. Rev. D 87 (2013) 052007.

[18] H. Kowalski and T. F. Walsh, Interference effects in J/ $\psi$ decay, Phys. Rev. D 14 (1976) 852.

[19] L. Kopke and N. Wermes, J/ $\psi$ decays, Phys. Rept. 174 (1989) 67.

[20] K. Zhu, X. H. Mo and C. Z. Yuan, Determination of the relative phase in $\psi^{\prime}$ and $J / \psi$ decays into baryon and antibaryon, Int. J. Mod. Phys. A 30 (2015) 1550148.

[21] M. Ablikim et al. (BESIIICollaboration), First observation of the isospin violating decay

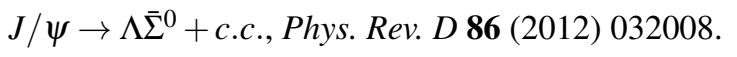

[22] B. L. Loffe, Masses of light quarks and interaction of low-energy $\eta$ mesons. (in Russian), Yad. Fiz. $29(1979) 1611$. 
[23] B. L. Ioffe and M. A. $\sim$ Shifman, Decays $\psi^{\prime} \rightarrow J / \psi \pi^{0}(\eta)$ and quark masses, Phys. Lett. B 95 (1980)

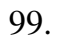

[24] Here we assume the $\eta$ is in the SU(3) octet, and the effect of the $\eta-\eta^{\prime}$ mixing is assumed to be small [RS].

[25] R. Casalbuoni, A. Deandrea, N. D1 Bartolomeo, R. Gatto, F. Feruglio and G. $\sim$ Nardulli, Hadronic Eransitions among quarkonium states in a soft exchange approximation. Chiral breaking and spin symmetry breaking processes, Phys. Lett. B 309 (1993) 163.

[26] E. K. Guo, C. Hanhart and U. G. $\sim$ Meissner, On the extraction of the light quark mass ratio from the decays $\psi^{\prime} \rightarrow J / \psi \pi^{0}(\eta)$, Phys. Rev. Lett. 103 (2009) 082003. Erratum: [Phys. Rev. Lett. 104 (2010] 109901

[27] M. Ablikim et al. (BESIIICollaboration), Precision measurements of branching fractions for $\psi^{\prime} \rightarrow \pi^{0} J / \psi$ and $\eta J / \psi$, Phys. Rev. $D \mathbf{8 6}$ (2012) 092008.

[28] M. Ablikim et al. (BESIII Collaboration), Measurements of $h_{c}\left({ }^{1} P_{1}\right)$ in $\psi^{\prime}$ Decays, Phys. Rev. Lett. $104(2010) 132002$.

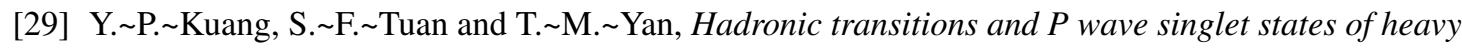
guarkonia, Phys. Rev. D 37 (1988) 1210.

[30] M. Ablikim et al. (BESIII Collaboration), Searches for isospin violating transitions $\chi_{c 0,2} \rightarrow \pi^{0} \eta_{c}$, Phys. Rev. D 91(2015) 112018 .

[31] L. Maiani, V. Riquer, R. Faccini, F. Piccinini, A. Pilloni and A. D. Polosa, $A J^{P G}=1^{++}$Charged Resonance in the $Y(4260) \rightarrow \pi^{+} \pi^{-} J / \psi$ Decay, Phys. Rev. D 87 (2013) 111102.

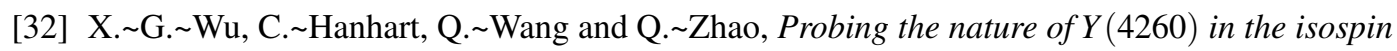
violating process $Y(4260) \rightarrow J / \psi \eta \pi^{0}$, Phys. Rev. D 89 (2014) 054038 .

[33] M. Ablikim et al. (BESIIICCollaboration), Search for the isospin violating decay $Y(4260) \rightarrow J / \psi \eta \pi^{0}$, Phys. Rev. D $92(2015) 012008$.

[34] E. van Beveren and G. Rupp, Observed $D(s)(2317)$ and tentative D(2030) as the charmed cousins of the light scalar nonet, Phys. Rev. Lett. 91 (2003) 012003.

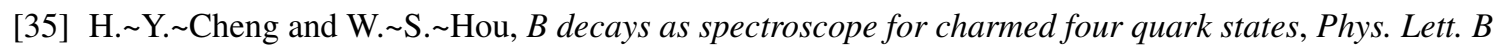
$566(2003) 193$.

[36] M. A. Nowak, M. Rho and I. Zahed, Chiral doubling of heavy light hadrons: BABAR 2317-MeV/c and CLEO 2463-MeV/c ${ }^{2}$ discoveries, Acta Phys. Polon. B 35 (2004) 2377.

[37] K. Terasaki, BABAR resonance as a new window of hadron physics, Phys. Rev. D 68 (2003) 011501.

[38] M. Ablikim et al. (BESIIICollaboration), Measurement of the absolute branching fraction of] $D_{s 0}^{* \pm}(2317) \rightarrow \pi^{0} D_{s}^{ \pm}$, Phys. Rev. $D 97$ (2018) 051103.

[39] M. Ablikim et al. (BESIII Collaboration), Observation of the electromagnetic doubly OZI-suppressed decay $J / \psi \rightarrow \phi \pi^{0}$, Phys. Rev. D 91 (2015) 112001. 